EPJ Web of Conferences 41, 12002 (2013)

DOI: $10.1051 /$ epjconf/20134112002

C) Owned by the authors, published by EDP Sciences, 2013

\title{
Self-referenced spectral interferometry for ultra-short infrared pulse characterization
}

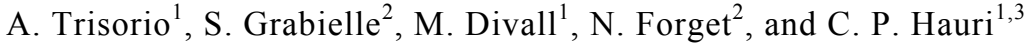 \\ ${ }^{1}$ Paul Scherrer Institute, SwissFEL, 5232 Villigen PSI, Switzerland \\ ${ }^{2}$ Fastlite, Centre Scientifique d'Orsay, Bat 503, Plateau du Moulon, BP45, 91401 Orsay, \\ France \\ ${ }^{3}$ Ecole Polytechnique Federale de Lausanne, 1015 Lausanne, Switzerland
}

\begin{abstract}
Self-Referenced Spectral Interferometry is used for single shot pulse characterization over the $0.9-2.5 \mu \mathrm{m}$ spectral range with a single spectrometer and an optimized optical setup. We characterize sub-55 fs pulses from $1.4 \mu \mathrm{m}$ to $2 \mu \mathrm{m}$ and broadband 2.5-cycle pulses at $1.65 \mu \mathrm{m}$ (13 fs FWHM).
\end{abstract}

The development of laser sources delivering tunable, ultra-broadband femtosecond pulses in the shortwavelength infrared (SW-IR) and mid-infrared (mid-IR) spectral ranges open-up the way for new laser pulse characterization devices. Recently developed laser systems make use of Optical Parametric amplification (OPA) [1] and Optical Parametric Chirped Pulse Amplification (OPCPA) [2] to produce sub-20 fs pulses covering bandwidth from 1.2 to $2.4 \mu \mathrm{m}$ with a few milli-Joule energy per pulse. A broadband, single shot characterization technique is mandatory, since these sources are based on nonlinear parametric frequency conversion, and so intensity fluctuations in the pump lead to shot to shot phase fluctuations in the signal. Up to our knowledge, none of the usually used characterization techniques (like FROG or SPIDER) have been adapted yet to few-cycle pulse characterization in the SWIR [3].

Self-Referenced Spectral Interferometry (SRSI) [4,5], discussed here, is a technique which offers intrinsically single-shot and frequency-conserving pulse reconstruction (i.e. the recorded signal is at the same wavelength as the input signal). It is thus possible to characterize both spectrum and phase with one single spectrometer. We demonstrate a SRSI based measurement device for ultra-short broadband SWIR pulse characterization with a single extended-InGaAS spectrometer. Single-shot measurement of sub-55 fs pulses in the 1.4 -2 $\mu \mathrm{m}$ spectral range is reported and attests to the broadband capability of the technique. In addition, we characterized few-cycle pulses ( $\left.13 \mathrm{fs} F W H M, \lambda_{0}=1.65 \mu \mathrm{m}\right)$ as an experimental confirmation of the device's capability to measure ultra-short pulses in the SWIR [3].

\section{Principle of SRSI and optical layout}

As shown in Fig. 1, Self-Referenced Spectral Interferometry [4,5] consists of analyzing the fringes of the interferogram created by mixing an unknown optical pulse $E(\omega)$ (pink arrows) and a reference pulse $E_{\text {ref }}(\omega)$ (blue arrows) delayed in time by $\tau$. In our case, the reference pulse is generated from fraction of the pulse to be measured passing through a temporal filter based on Cross-Polarized Wave Generation (XPW) [6], a degenerate third-order nonlinear process. It will be denoted as $E_{X P W}(\omega)=E_{\text {ref }}(\omega)$. The interferometric signal measured by the spectrometer can be expressed in the form:

This is an Open Access article distributed under the terms of the Creative Commons Attribution License 2.0, which permits unrestricted use, distribution, and reproduction in any medium, provided the original work is properly cited. 


$$
S(\omega)=\left|E_{X P W}(\omega)+E(\omega) e^{i \omega \tau}\right|^{2}=S_{0}(\omega)+2 \operatorname{Re}\left[g(\omega) e^{i \omega \tau}\right]
$$

Where $S_{0}$ is the sum of the input and XPW pulses spectra and $g$ the interference term between these two pulses. Both spectral amplitudes $E(\omega)$ and $E_{X P W}(\omega)$, are directly reconstructed by Fourier Transform Spectral Interferometry (FTSI) [7].
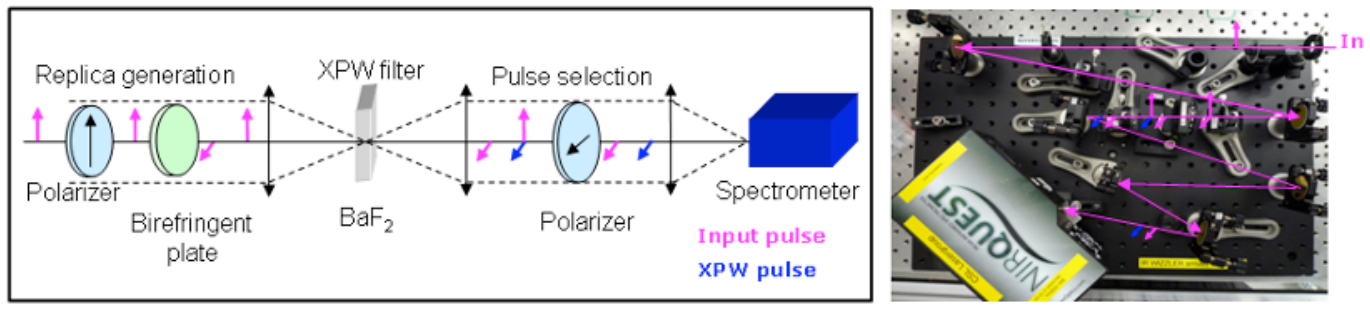

Fig. 1. Left: schematic and right: picture of the SRSI measurement device.

In the optical setup, a high-contrast reflective polarizer defines the polarization direction of the input pulse $\mathrm{E}(\mathrm{t})$ (pink arrow in the paper plane in Fig. 1). This pulse is focused in a [100]-oriented $\mathrm{BaF}_{2}$ crystal (transparent over $0.15-12 \mu \mathrm{m}$ ). The orthogonally polarized pulse $\mathrm{E}_{\mathrm{xpw}}(\mathrm{t})$, generated by $\mathrm{XPW}$, is selected by a second polarizer oriented as analyzer (blue arrow, Fig. 1). To perform spectral interferometry, a $0.5 \mathrm{~mm} \mathrm{YVO}_{4}$ birefringent plate is inserted before the $\mathrm{BaF}_{2}$ crystal to generate an orthogonally polarized replica of the input pulse (red arrows oriented perpendicularly to the paper plane), which interferes with the XPW pulse in the spectrometer (OceanOptics NIRQuest512-2.5 with an optical resolution of $6.3 \mathrm{~nm}$ at $1.5 \mu \mathrm{m}$ ). For broadband and dispersion-free operation, steering and focusing optics are Au-coated mirrors. Our SWIR optical source consists of a commercial OPA delivering sub-55 fs pulses at $100 \mathrm{~Hz}$ in the $1.2-2 \mu \mathrm{m}$ spectral range. For few-cycle operation, the OPA output is focused in a gas cell filled with Xenon ( $\mathrm{P}=2.5$ bar) to spectrally broaden the pulse by filamentation. The same SRSI setup was used to characterize both the OPA and the few-cycle pulses.

\section{Experimental results}

We performed single-shot SRSI measurements of the OPA output pulses [3]. Fig. 2 shows the reconstructed pulses for different central wavelength over the OPA's available bandwidth. After residual dispersion compensation, all pulses have a measured duration (FWHM) that is less than 5\% longer than the Fourier limited one. A consistency check for the measurement was performed to verify that the XPW spectrum was broader and the FL duration shorter than those of the pulse to be measured. The device allows to perform accurate and user-friendly single-shot characterization of the compressed pulses over the full 1.4-2 $\mu \mathrm{m}$ spectral range.

In Fig. 3, one can see the SRSI characterization of ultra-broadband few-cycle pulses generated by filamentation at $1500 \mathrm{~nm}$ [8]. Pulse durations down to 13.6 fs FWHM (red) could be measured with a small deviation of 0.9 fs with respect to the FL (12.5 fs FWHM, black dashed). The corresponding spectrum is depicted in Fig. 3 (b) (red): the bandwidth is equal to $742 \mathrm{~nm}$ (at $1 \%$ of the maximum intensity). The measured spectral phase (Fig. 3 (b), blue squared line) is quasi-flat. We calculated the measurable temporal contrast level (limited by the spectrometer noise) to be $55 \mathrm{~dB}$. 

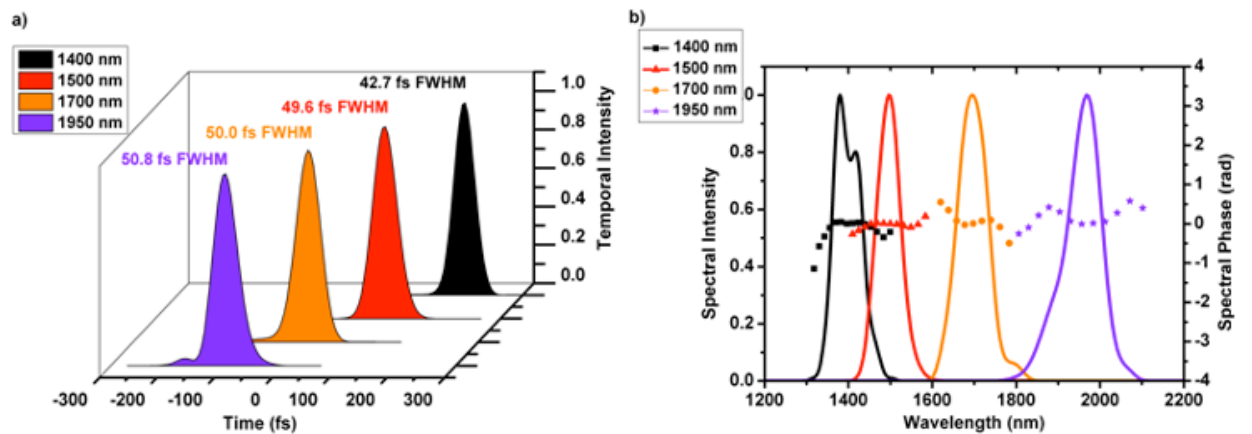

Fig. 2. Measured: a) pulse temporal profiles, b) spectra (solid lines) and spectral phases (symbols) at the OPA output at $1400 \mathrm{~nm}$ (black), $1500 \mathrm{~nm}$ (red), $1700 \mathrm{~nm}$ (orange) and $1950 \mathrm{~nm}$ (purple). All measurements are single shot. The $\mathrm{BaF}_{2}$ crystal thickness was $2 \mathrm{~mm}$.
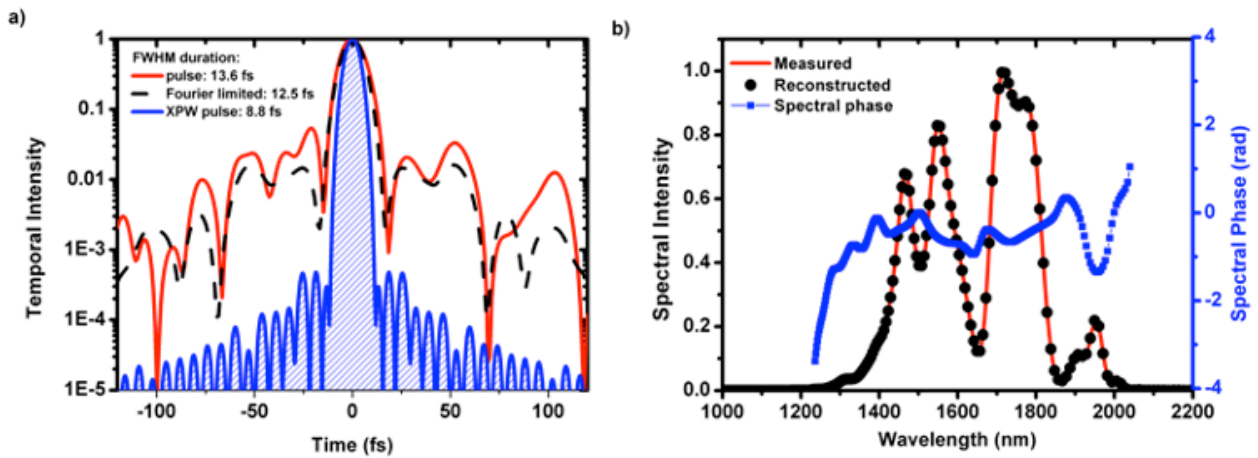

Fig. 3. a) Single-shot reconstructed temporal profile of the $13.6 \mathrm{fs}$ FWHM pulse (red line) on a logarithmic scale. The Fourier limit is 12.5 fs FWHM (black line). The retrieved XPW FL pulse is shown in ble. b) Measured (red line) and reconstructed (black dots) normalized spectra and spectral phase (blue squares) of the compressed pulse. The $\mathrm{BaF}_{2}$ crystal thickness was $1 \mathrm{~mm}$.

In conclusion, we applied for the first time Self-Referenced Spectral Interferometry for characterization of broadband, few-cycle pulses in the short-wavelength mid-infrared spectral range [0.9-2.5 $\mu \mathrm{m}]$. Pulses as short as $13.6 \mathrm{fs}$ FWHM could be measured. The optical setup and pulse retrieval algorithm are optimized for single-shot pulse characterization with a high $55 \mathrm{~dB}$ dynamic range in the temporal domain.

\section{References}

1. C. Vozzi, F. Calegari, E. Benedetti, S. Gasilov, G. Sansone, G. Cerullo, M. Nisoli, S. De Silvestri and S. Stagira, Opt. Lett. 32, 2957 (2007)

2. O. D. Mucke, S. Alisauskas, A. J. Verhoef, A. Pugzlys, A. Baltuska, V. Smilgevicius, J. Pocius, L. Giniunas, R. Danielius, N. Forget, Opt. Lett. 34, 2498 (2009)

3. A. Trisorio, S. Grabielle, M. Divall, N. Forget and C. P. Hauri, Opt. Lett. 37, 2892 (2012)

4. T. Oksenhendler, S. Coudreau, N. Forget, V. Crozatier, S. Grabielle, R. Herzog, O. Gobert, D. Kaplan, Appl. Phys. B 99, 7 (2010)

5. S. Grabielle, A. Moulet, N. Forget, V. Crozatier, S. Coudreau, R. Herzog, T. Oksenhendler, C. Cornaggia, O. Gobert, Nucl. Inst. and Meth. in Phys. Res. A 653, 121 (2011)

6. N.Minkovski, G.I. Petrov, S.M. Saltiel, O.Albert, J. Etchepare, JOSA B 21, 1659 (2004)

7. L, Lepetit, G, Cheriaux, M. Joffre, JOSA B 12,2467 (1995) 